\title{
Ulcer Piercing: A Novel Drainage Technique for Diabetic Patients with Complicated Foot
}

\author{
Cavallini Marco* \\ Director of Surgical Unit, Director of Master on Wound Care, Faculty of Medicine and Psychology, Department of Medical-Surgical Sciences and \\ Translational Medicine, Sapienza, University of Rome, AO Sant'Andrea, Via di Grottarossa, Via di Grottarossa, 1035-1039, 00189 Rome, Italy
}

Received: July 07, 2015; Accepted: August 15, 2015; Published: September 11, 2015

*Corresponding author: Cavallini Marco, Director of Surgical Unit, Director of Master on Wound Care, Faculty of Medicine and Psychology, Department of Medical-Surgical Sciences and Translational Medicine, Sapienza, University of Rome, AO Sant'Andrea, Via Pompeo Magno Via di Grottarossa, 1035-1039, 00189 Rome, Italy, Tel: +39-3355267267; E-mail: marco.cavallini@uniroma1.it

\begin{abstract}
To achieve ulcer healing of diabetic foot, to avoid any fluid or exudate stasis, surgical drainage of any superficial or deep recess is mandatory. We have designed a surgical procedure of ulcer piercing (UP) and drainage with silastic tube which allows a continuous or daily positive pressure irrigation of any pierced hidden tract. This procedure has been utilize in a continuous series of 35 selected diabetic patients affected by a Wagner stage 3 ulcer of a toe $(n=19)$, metatarsal midfoot $(n=10)$ and plantar Charcot foot $(n=6)$ and with an adequate foot arterial blood supply. 33/35 ulcer have completely healed within 7 months of ulcer piercing procedure while 2 cases of acute Charcot foot after being cleared their infection condition underwent below the knee amputation. Therefore, keeping in mind the advantages and considering the lack of adverse or side effects or contraindications, in our opinion, the Ulcer Piercing (UP) procedure represents a small but effective step toward a better, easier and safer approach in the treatment of complicated diabetic neuropathic foot ulcers with adequate arterial blood supply.
\end{abstract}

Keywords: Wound healing; Diabetic foot; Foot infection; Foot ulcer

\section{Introduction}

Diabetic foot ulcers present a great challenge to wound care practitioners. Diabetic foot, complicated by a deep contaminated neuropathic ulcer, is quite often observed in the hospital's emergency rooms or in the surgical outpatient's facilities. This condition is mainly the consequence of inadequate prevention programs on the territory and/or inadequate and ineffective procedures to avoid extension of any superficial wounds to progress into the underlining deeper spaces eventually involving the bones. The clinical problem is serious since these conditions can progress to a more critical condition which can jeopardize the integrity of foot stability leading to less or wider extended radical interventions. It has been documented, in a total of 917 diabetic patients, that ulcer healing is an independent predictor of patient survival and amputation-free survival [1].

Diabetic foot ulcer infection is a surgical complication which requires adequate ulcer debridement, resection of all dead/infected tissues, effective drainage and systemic specific antibiotics. Surgical drainage of any superficial or deep recess of the ulcer is mandatory to avoid any fluid or exudate stasis: stasis is the main favorable condition for persistent bacterial contamination and infection. Therefore, in order to reduce the negative impact of stasis and critical bacterial contamination on wound healing, we have, recently, designed a simple surgical procedure of Ulcer Piercing (UP) and drainage which allows a constant adequate cleansing of the ulcer and facilitates irrigation of any pierced hidden tract. We have successfully utilized this technique in a series of diabetic patients, affected by deep complicated neuropathic foot ulcers [2] and in other settings of complicated deep cutaneous ulcers such as pressure ulcers, perianal abscesses, post-traumatic soft tissue lesions and surgical wound dehiscence [3].

\section{Materials and Methods}

A continuous series of 35 diabetic selected patients, 19 female and 16 male, mean age $59 \pm 8$ years old, affected by complicated foot with a deep, Wagner stage 3, neuropathic infected ulcer, have been recruited in this study between May 2011 and July 2014. Limb's arterial blood supply was considered as adequate in the presence of peripheral (tibial) arterial pulses or with $\mathrm{ABI}>$ 0.6 and/or $\mathrm{TcPO}_{2}>30 \mathrm{mmHg}$.

Patients' consent to UP procedure was obtained for this conservative procedure as an alternative of surgical drainage with extended incision of the hidden tracts we usually utilize in these cases.

Deep contaminated ulcers were localized in the lateral interdigital surface in 8 patients (Figure 1) and in the dorsal digital surface in 11 instances. Deep contaminated tract between a toe ulcer and the respective metatarsal abscess has been observed in 10 patients (Figure 2). Deep contaminated ulcer of the plantar surface has been treated in 6 patients with a complicated Charcot foot. In all instances wound exploration with a probe was carried out and, when indicated, any exposed infected bone and/or infected tendon removed (Figure 3). Under local anesthesia, at the opposite site of any main recess opening, where the end of the tract become superficial toward the skin and in the more depen- 


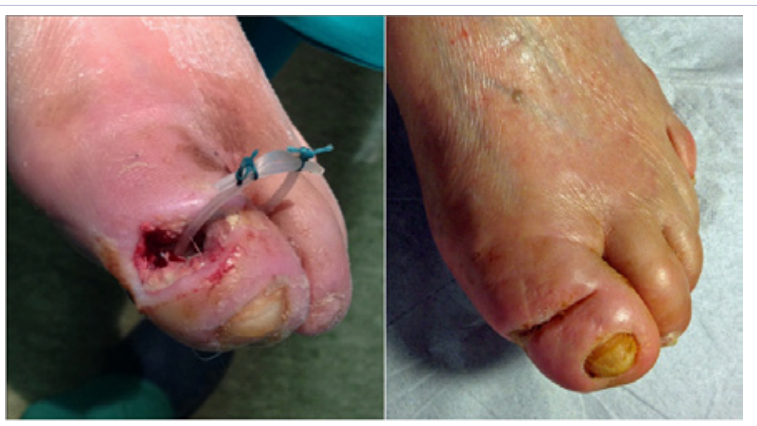

Figure 1: A patient with the arterial obstructive disease of the lower limbs and osteomyelitis of the proximal phalanx of the first toe treated with an ulcer piercing (UP) ring (left). The ulcer healed 3 months later (right).

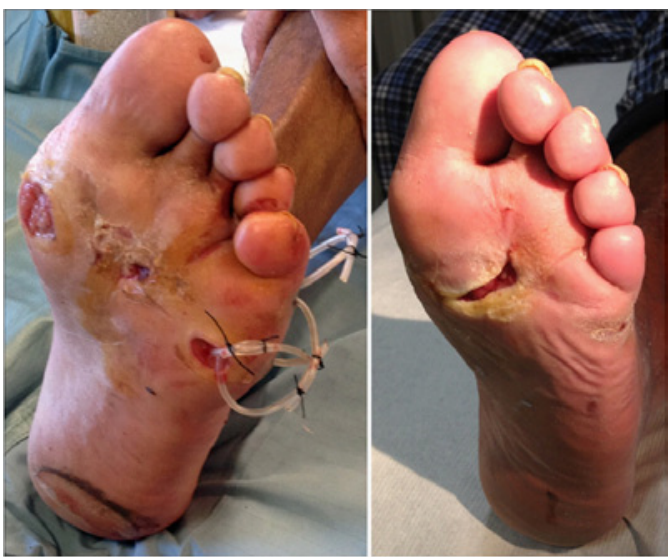

Figure 2: Infection of the 5th metatarsal head treated with multiple ulcer piercing rings (left), both 1st and 5th metatarsal ulcers have healed while the central plantar ulcer has required an extension of the surgical incision to allow a better cleansing.

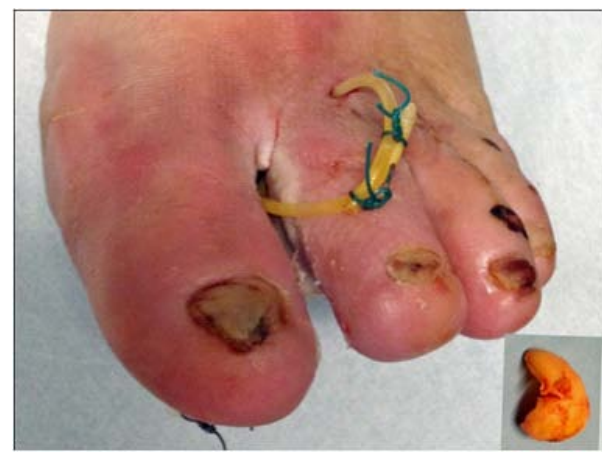

Figure 3: A patient with a deep infection of the 2nd metatarsal head treated with an ulcer piercing ring after the removal of the infected metatarsal head articular surface (see box).

dent site according to patient preferred decubitus, interposed tissues and the skin are pierced and incised in order to pass through the probe and, subsequently, a small soft silastic tube. The silastic tube is anchored to the probe with a stitch in order to pass it backward along the fistula tract. In the early experience, we have utilized the tube of a butterfly needle. Once this drain- age is passed along the ulcer's recess tract, the two ends are tied together with two silk stitches in order to construct a blocked ring (UP ring) (Figure 4). The UP ring, therefore, is designed to keep the tract open and to facilitate the insertion of a syringe into both sides of the openings (Figure 5) and facilitate fistula flash. The patient, the family members, and the home caregiver are then instructed and invited to learn how to irrigate, twice a day with a syringe, the UP tract with a saline or Dakin preparation. Dakin solution is preferred in cases with clinical signs of active infection such as exudates, pain and edema, perilesional erythema. Interesting enough, is the fact that family members and non-professional care givers, in general, do feel more comfortable in dealing with a "piercing procedure" than with a "surgical drainage" since nowadays piercing rings have been accepted as a fashion procedure. Subsequently, all patients have been followed in the Hospital outpatient's facility once a week or every two weeks. The UP-ring has been replaced once a week in the presence of clinical active signs of infection (edema, perilesional erythema, pain, exudate, etc.) or once every two weeks in order to evaluate with a probe the growth of granulation tissue and the covering process of ring's tract or of the exposed bones. The UPring is definitely removed once the tract kept open by the ring is completely covered by granulation tissue (Figure 6). All patients have been treated with systemic specific antibiosis on the basis of the results of bed ulcer's culture and its relative antibiotic assay. Intramuscular or intravenous (the latter in the case of hospitalized patients) treatment with teicoplanin and cefepime or meropenem was the main antibiotic association in the early treatment of any deeply infected ulcer. In case of outpatient ambulatory setting, as oral antibiosis, quinolone drugs (levofloxacin, ciprofloxacin or moxifloxacin) were prescribed, as a first choice, for 2 to 3 week period treatment. As pressure relief systems, patients with forefoot ulcer have utilized half-shoes while in cases of complicated Charcot foot a removable cast walker with air cell system has been indicated. In any cases, patients have been invited to reduce their walking activity up to the healing process was started.

\section{Results}

33/35 (94\%) ulcer have healed within 7 months after the UP procedure was performed and in no cases an adverse or side effects of UP ring has been observed. In all patients, the Up ring has been definitely removed only when the pierced tract was homogeneously covered by granulation tissue and no more exposed bones were detected by probe tract exploration. In 19 patients with toe ulcer healing has been achieved in a range time between 3 and 5 months, 10 patients with a metatarsal/plantar abscess healed between 4 and 7 months (Figure 7). 4 patients with a Charcot's foot and plantar ulcer have healed between 4 and 6 months. The other 2 patients affected by acute Charcot foot, with no longer active signs of infection, underwent below the knee amputation.

\section{Discussion}

Inflammation is a physiological response to wounding and represents, after an acute injury, the early phase of wound 

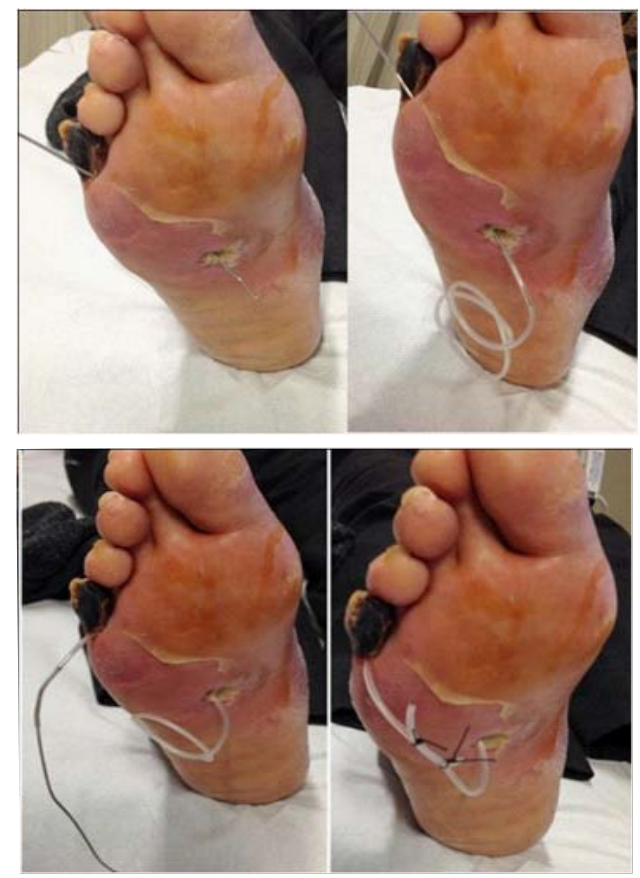

Figure 4: The opposite the site of the main ulcer recess, where the end of the tract is more superficial toward the skin and in the more dependent site according to patient preferred decubitus, interposed tissues and the skin are pierced and incised in order to pass through the probe which facilitates to pass backward the silastic tube along the pierced tract.

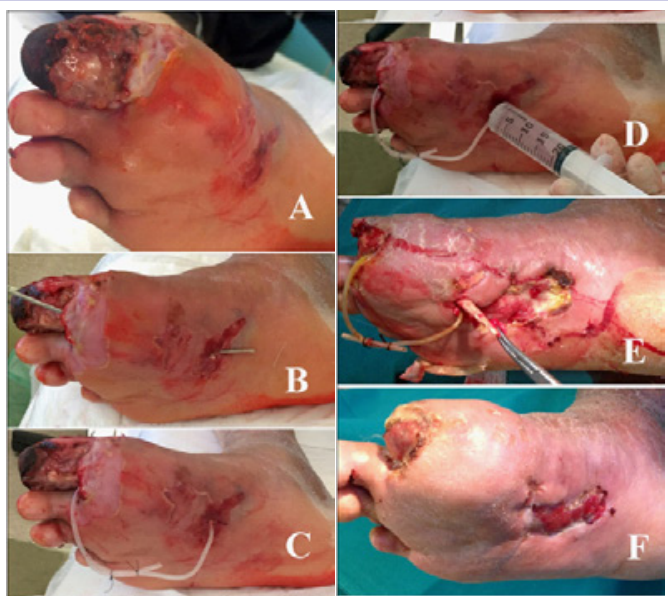

Figure 5: The Ulcer Piercing ring is designed to keep the tract open and to facilitate the insertion of a syringe into both sides of the openings for ulcer tract cleansing. A: Complicated diabetic foot with ulcer of the first toe and a fistula tract developed along the flexor tendon and cause of the plantar space abscess. B: Under local anesthesia, at the opposite site of the fistula tract where it becomes superficial the skin is pierced and incised in order to pass through the probe. C: The silastic tube is passed backward and the ring structured and blocked with two silk stitches, D: The ring, therefore, is designed to keep the tract open and to facilitate the insertion of a syringe into both sides of the openings and facilitate fistula irrigation and cleansing. E: The necrotic/infected distal end of the first toe has been removed together with the infected flexor tendon. F: The tract kept open by the ring is going to heal after removal of the drainage.
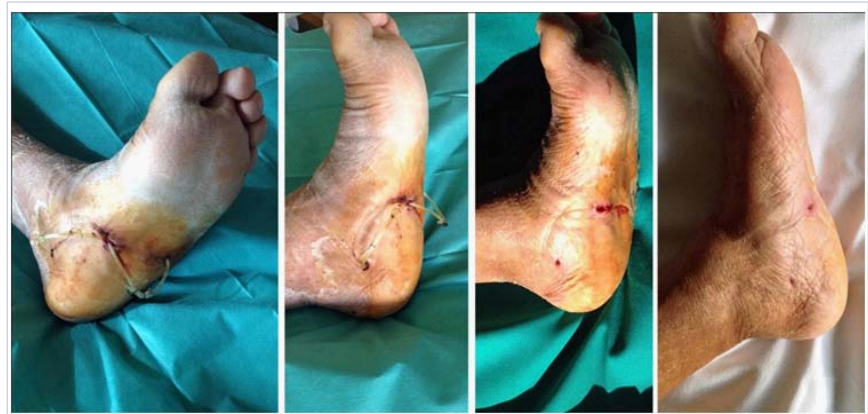

Figure 6: The Ulcer Piercing ring is definitely removed once the tract kept open by the ring is completely covered by granulation tissue leading to ulcer closure.

healing. Excessive inflammation due to the persistence of a critical bacterial contamination, biofilm or infection leads to wound chronicity [4]. Stalling of ulcer healing, which do not progress beyond the inflammatory phase, has been related to persistent inflammation [5] and an increase of matrix metalloproteases and elastase activities [6,7]: Chronic degradation of extracellular matrix, suppression of growth factors function, an increase of pro-inflammatory cytokines (TNF- $\alpha$, IL-1, IL-6) [8], which inhibit down-regulation of the immune response, and the prevalence of senescent fibroblasts at the end of their cell cycle [9] further hinder wound healing. Any local or systemic treatment finalized to eliminate or reduce prolonged inflammation, therefore, revitalizes physiological tissue healing, reduces exudate and is associated with a reduction in bioburden.

On this regard, the results of a study by Alfano, et al. [10], demonstrating the effectiveness of careful surgical debridement and vacuum therapy in improving and accelerating ulcer healing, are of particular interest. More specifically, vacuum therapy results in a continuous aspiration of bed ulcer, therefore, reducing the possibility of ulcer fluid stasis and critical bacterial contamination/infection. This procedure seems to be particularly useful and effective in cases with diabetic foot ulcers [11].

Therefore, to achieve ulcer healing, in patients with adequate tissue arterial blood supply underlying, surgical debridement of ulcer bed and removal of all infected/dead tissues are mandatory along with drainage gauze of all ulcer recess or fistulas and systemic antibiotics. In cases of a deep narrow ulcer recess or fistula, surgical drainage could consist in an extended incision of the tract and in the apposition of a draining gauze, Penrose or rubber drain. These latter solutions, however, are not always effective and safe and could be detrimental since they can become obstructive or can be easily and/or accidentally removed during home care treatments. Moreover, quite often, any small skin wound, fistula or incision progressively reduces its opening, therefore interfering with ulcer tract drainage. In the case of a deep and narrow, not well drained ulcer recess UP drainage procedure, in our opinion, is useful to warrant effective tract cleansing by daily positive pressure irrigation (Figure 5). UP procedure is easy and safe and the constructed blocked ring of tubing system avoids the possibility for drainage displacement or accidental removal. This procedure increases the ease at which 


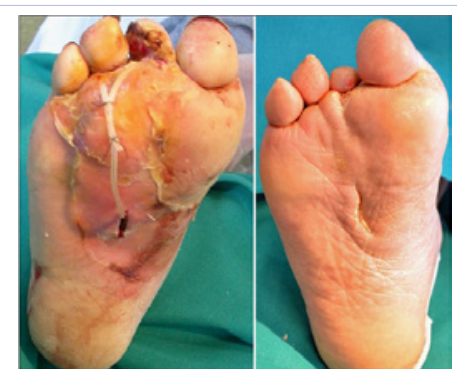

Figure 7: A patient with a deep infection of the metatarsal mid-foot originated from the respective ulcerated toe, treated with an ulcer piercing ring after the removal of the infected flexor tendon and completely healed after 4, 5 months avoiding a wider, full tract length, incision and a longer plantar scar.

the patients themselves or the caregivers (nurses or family) can perform daily cleansing and wound care in the home care setting. In our opinion, this conservative technique should be considered as a first step procedure in treating not well-drained ulcer recesses because it could avoid unnecessary and untimely extended and deep tissue incisions which, as a consequence, can result in scarring.

\section{Conclusions}

To achieve cutaneous ulcer healing, in order to avoid any fluid or exudate stasis, we have designed a novel procedure involving ulcer piercing and drainage of any hidden tract and daily positive pressure irrigation and cleansing. Since ulcer debridement and drainage are a vital adjunct in the care of patients with chronic diabetic foot ulcers, in our opinion, this procedure of ulcer piercing drainage represents a small but effective step towards an easier and safer treatment of cutaneous ulcers with not welldrained deeper recesses.

\section{References}

1. Taylor SM, Johnson BL, Samies NL, Rawlinson RD, Williamson LE, Davis SA, et al. Contemporary management of diabetic neuropathic foot ulceration: a study of 917 consecutively treated limbs. J Am Coll Surg, 2011;212(4):532-545.

2. Cavallini M. Ulcer piercing: cleansing of complicated diabetic neuropathic foot ulcers by positive pressure irrigation. J Wound Care. 2014;23(2):60-65.

3. Cavallini M. Wound piercing: a novel approach for deep cutaneous ulcer cleansing. Ann. Ital. Chir. 2014;85(5):506-510.

4. Percival SL, Hill KE, Williams DW, Hooper SJ, Thomas DW, Costerton JW. A review of the scientific evidence for biofilms in wounds. Wound Repair Regen. 2012;20(5):647-57. doi: 10.1111/j.1524475X.2012.00836.x.

5. Dechert TA, Ducale AE, Ward SI, Yager DR. Hyaluronan in human acute and chronic dermal wounds. Wound Repair Regen. 2006;14(3):252258.

6. Dinh T, Tecilazich F, Kafanas A, Doupis J, Leal E, Tellechea A. Mechanisms involved in the development and healing of diabetic foot ulceration. Diabetes. 2012;61:2937-47. doi: 10.2337/db12-0227.

7. McCarty SM, Cochrane CA, Clegg PD, Percival SL. The role of endogenous enzymes in chronic wounds: a focus on the implications of aberrant levels of both host and bacterial proteases in wound healing. Wound Repair Regen, 2012;20(2):125-36. doi: 10.1111/j.1524475X.2012.00763.x.

8. Trengove NJ, bielefeldt-Ohmann H, Stacey MC. Mitogenic activity and cytokine levels in non-healing and healing chronic leg ulcers. Wound Repair Regen. 2000;8:13-25.

9. Agren MS, Steenfos HH, Dabelsteen S, Hansen JB, Dabelsteen E. Proliferation and mitogenic response to PDGF-BB of fibroblasts isolated from chronic venous leg ulcers is ulcer-age dependent. J Invest Dermatol. 1999;112(4):463-469.

10. Alfano C, Angelisanti M, Calzoni C, Somma F, Chiummariello S. Treatment of ulcer and difficult wounds of the lower limbs: our experience. Ann Ital Chir. 2012; 83:135-41.

11. Lone AM, Zaroo MI, Laway BA, Pala NA, Bashir SA, Rasool A. Vacuumassisted closure versus conventional dressing in the management of diabetic foot ulcers: a prospective case-control study. Diabetic Foot Ankle. 2014;8;5. eCollection 2014. doi: 10.3402/dfa.v5.23345. 